\title{
A delay-specific differential outcomes effect in delayed matching to sample
}

\author{
K. Geoffrey White • Rebecca J. Sargisson
}

Published online: 18 March 2015

(C) Psychonomic Society, Inc. 2015

\begin{abstract}
In delay-specific remembering, accuracy in delayed matching-to-sample tasks is enhanced after single delays or retention intervals relative to performance at other delays. In the differential-outcomes effect (DOE), accuracy is enhanced at all delays when the outcomes of correct choices are quantitatively or qualitatively different, compared to when outcomes are the same. In the present experiments, we aimed to demonstrate a delay-specific DOE by arranging differential outcomes for correct responses at some delays and same outcomes at other delays. In each of two experiments, four pigeons worked in delayed matching-to-sample tasks with delays of $0.5,5$, and $15 \mathrm{~s}$, or $0 \mathrm{~s}, 3 \mathrm{~s}$, and $12 \mathrm{~s}$ mixed within session. Correct choices produced different reward durations (differential outcomes) at one or two delays, or the same reward durations (same outcomes) at the other delays, on a within-session basis. There was evidence of improved accuracy at delays at which differential outcomes were arranged, compared to accuracy at delays at which same outcomes were arranged, that is, a delay-specific DOE. The more usual DOE was confirmed in a third experiment with same outcomes at all delays in one condition and differential outcomes at all delays in another. We discuss implications of a delay-specific DOE for theories of the DOE which attribute the effect to enhanced stimulus control by expectancies of reward outcomes generated at the time of sample presentation.
\end{abstract}

Keywords Differential-outcome effect · Delay-specific remembering $\cdot$ Delayed matching to sample $\cdot$ Pigeon

K. G. White $(\triangle) \cdot$ R. J. Sargisson

Department of Psychology, University of Otago, Dunedin,

New Zealand

e-mail: geoff.white@otago.ac.nz

K. G. White $\cdot$ R. J. Sargisson

University of Waikato, Hamilton, New Zealand
Remembering over relatively short times is often studied in delayed matching-to-sample (DMTS) tasks, in which reinforcement follows a response to a comparison stimulus that matches one presented before a retention interval, or delay. Typically, the accuracy of the animal's choice decreases as the delay is lengthened (White, 1985, 2001, 2013; Wixted, 1989). However, it is not always the case that performance worsens with lengthening delay, as demonstrated by White and Brown (2011a, b). In one set of experiments, White and Brown (2011a) reported forgetting functions that increased at intermediate delays by removing retroactive interference (turning off the houselight) partway through longer delays. In another experiment, White and Brown (2011b) used cues to signal the availability of a small or large reinforcer for correct comparison choices. Accuracy improved on trials when a large reinforcer was signaled (the signaled magnitude effect). But when the small-reinforcer cue was switched to the cue for the large reinforcer partway through the delay, accuracy improved with longer delays from the time at which the cue was reversed. The reversed forgetting functions in these experiments are consistent with the notion that remembering can be specific to the delay at which remembering occurs (White, 2002, 2013).

In a further example of delay-specific remembering, Sargisson and White (2001) trained different groups of pigeons with single non-zero delays from the outset of DMTS training. In subsequent probe tests with mixed delays, accuracy was higher at the training delay than at shorter or longer delays. Similarly, comparison choices at one delay within sessions with mixed delays can be made more (Nakagawa, Etheridge, Foster, Sumpter, \& Temple, 2004) or less (White, 2001) accurate by including or omitting reinforcement following that delay. Yet other indications of delay-specific remembering are provided by the results of probe tests in which signals cueing short and long delays during DMTS training are reversed (McDonald \& Grant, 1987; Wasserman, Grosch, 
$\&$ Nevin, 1982), and by reversal of the reinforcement contingency between short and long delays in order to generate an explicit dependence of accurate remembering on whether the delay is long or short (Sargisson \& White, 2007; White \& Sargisson, 2011).

Our aim in the present experiments was to examine the possibility of a delay-specific differential outcomes effect (DOE). The DOE is manifest as enhanced accuracy when different outcomes are arranged for correct choices in DMTS, compared to accuracy with same outcomes for correct choices. This well established effect has been demonstrated in a range of species including pigeons (e.g., Nevin, Ward, Jiminex-Gomez, Odum, \& Shahan, 2009; White \& Millar, 2014), dogs (Overmier, Bull, \& Trapold, 1971), rats (Savage \& Parsons, 1997; Trapold, 1970), horses (Miyashita, Nakajima, \& Imada, 2000), and humans (Estevez, Overmier, \& Fuentes, 2003; Legge \& Spetch, 2009). Outcomes may differ in quantity or quality, or in other ways such as reinforcer location (Williams, Butler, \& Overmier, 1990). For example, in a recent study by Friedrich and Zentall (2011), pigeons chose between red and green comparison stimuli, and correct choices resulted in food being delivered in different spatial locations depending on whether samples were red or green. Accuracy was higher at all delays compared to accuracy for a group of pigeons for whom the different spatial locations were not correlated with sample hue.

In DMTS tasks with delays of varying duration, accuracy is typically enhanced by differential outcomes to a greater extent at long delays than at short delays, compared to when choice responses produce the same outcome (Brodigan \& Peterson, 1976; De Long \& Wasserman, 1981; Jones \& White, 1994; Peterson, Linwick, \& Overmier, 1987; Santi \& Roberts, 1985; Urcuioli, 1990; White \& Millar, 2014). In such studies, differential or same outcomes remain unchanged across the different delays. If, within session, differential outcomes were arranged after some delays, and same outcomes after other delays, it might be possible to improve accuracy after those delays associated with differential outcomes relative to delays associated with same outcomes. Such a result would be consistent with a delay-specific DOE.

We report two experiments which use the DMTS task with pigeons, and with three delays; short, intermediate, and long. In both experiments, outcomes were same or different durations of food presentation, and could be same or different at any one of the delays on a within-session basis. Because the delays were randomly mixed within session, the pigeon could not predict whether a particular trial would end in differential outcomes (e.g., at the intermediate delay) or same outcomes (e.g., at the short delay). For example, compared to a condition in which same outcomes were arranged at the intermediate delay, a condition with different outcomes at the intermediate delay but same outcomes at the short delay might yield enhanced accuracy at the intermediate delay, consistent with a delay-specific DOE. We also report a third experiment which confirmed the occurrence of the DOE for the present version of the DMTS task.

A delay-specific DOE is of potential theoretical interest. Urcuioli (2005) reviewed substantial evidence for the view that the DOE involves hypothetical reward expectancies, as originally envisaged by Trapold (1970). An expectancy of a given outcome is developed at the time of sample presentation and provides an additional source of stimulus control over choice responding to enhance accuracy. Indeed, Holden and Overmier (2014) recently showed that expectancies in differential outcome conditions may exert even greater stimulus control than the sample stimuli themselves. In the present experiments, however, trials ending in same outcomes at some delays were randomly mixed with trials ending in different outcomes. Owing to the unpredictability of whether outcomes were same or different, outcome expectances of the kind generated in typical differential-outcome conditions are unlikely to occur at the time of sample presentation in the present experiments. A delay-specific DOE would therefore not necessarily be anticipated by the outcome-expectancy theory of the DOE.

\section{Experiment 1}

In Experiment 1, we trained pigeons in a DMTS task with delays of $0.5,5$, and $15 \mathrm{~s}$ mixed within session. In the Same-Different-Same (SDS) condition, the outcome for correct choices was the same after 0.5 -s and 15 -s delays, and different after 5-s delays. In the Different-Same-Different (DSD) condition, the outcome for correct choices was different after 0.5 -s and 15-s delays and the same after 5-s delays. Each condition was replicated in order to balance for possible order effects. The between-condition comparisons of interest were at each of the delays, because at each delay a DOE was possible, independently of a DOE at each other delay.

\section{Method}

Subjects Four adult homing pigeons (Columba livia), $\mathrm{H} 1$ to $\mathrm{H} 4$, with prior experience in DMTS tasks, lived in individual cages with free access to water and grit in a colony room with a 12-h:12-h light:dark cycle supplemented by natural light. The pigeons were maintained at between 80 and $85 \%$ of their free-feeding body weights by supplementary feeding of mixed grain at the end of the daily session.

Apparatus Each of four experimental chambers was $31 \mathrm{~cm}$ wide, $34 \mathrm{~cm}$ deep, and $32 \mathrm{~cm}$ high. Three $2-\mathrm{cm}$ diameter response keys were positioned $10 \mathrm{~cm}$ apart on one wall, with a central opening beneath that allowed access to wheat as a reinforcer. The chamber was painted matte black, and was 
dark during sessions, except when the keys were illuminated red or green, or during reinforcer delivery when the hopper was lit. A ventilation fan at the rear of the chamber helped to mask extraneous sounds. Experimental events were controlled and recorded by a computer running MedPC software and interfacing in an adjacent room.

Procedure Owing to about 2 years of prior experience in DMTS with red and green samples and short delays, preliminary training was unnecessary and the pigeons proceeded directly to Experiment 1 . Each daily session of 96 trials began with the center key illuminated red or green. Five pecks on the center key initiated a delay of $0.5,5$, or $15 \mathrm{~s}$, after which one of the side keys was lit red and the other green. Sample colors (red or green), delay length, and comparison side colors (red and green on left and right keys) were chosen randomly trial by trial in blocks so that each sample was paired with each comparison arrangement and with each delay equally often. A single peck to either left or right comparison key produced a reinforcer delivery, or initiated a 12-s inter-trial interval (ITI). When the color of the comparison key pecked by the pigeon matched the color presented as the sample, reinforcement was made available prior to the ITI. On same-outcome trials, 3-s access to wheat served as a reinforcer for both correct red and correct green choices. On different-outcome trials, correct red choices produced 1.5-s access to wheat, and correct green 4.5$\mathrm{s}$ access to wheat. Reinforcer duration was timed from the comparison key peck. A new trial began after the ITI.

In the SDS condition, same-outcome trials were programmed for delays of 0.5 and $15 \mathrm{~s}$, while different outcomes followed correct comparison choices after 5-s delays. In the DSD condition, same-outcome trials were programmed for the 5-s delays, while correct comparison choices after delays of 0.5 and $15 \mathrm{~s}$ led to different outcomes. Conditions were run in the order: (1) SDS (38 sessions for each pigeon), (2) DSD (38 sessions for each pigeon), (3) DSD (44 sessions for H1 and $\mathrm{H} 4 ; 28$ sessions for $\mathrm{H} 2$ and $\mathrm{H} 3$ ), and (4) SDS (44 sessions for each pigeon).

Data analysis Correct (responses to the matching comparison key) and incorrect (or error; responses to the non-matching comparison key) choices from the last five sessions of each condition were summed for each pigeon at each delay. Accuracy, or discriminability, was measured as

$\log d=0.5 \cdot \log \left(\left(\frac{c_{\text {red }}}{e_{\text {red }}}\right)\left(\frac{c_{\text {green }}}{e_{\text {green }}}\right)\right)$

which is the log (base 10) of the geometric mean of the ratios of correct $(c)$ to error $(e)$ responses following the red and green samples (Davison \& Tustin, 1978; White, 1985). Unlike proportion correct, $\log d$ is not bounded by 1.0 (Nevin \& Grosch, 1990), and theoretically is unaffected by response bias
(Davison \& Tustin, 1978). In order to avoid infinite $\log d$ values in instances where no errors were made, 0.25 was added to each response total as recommended by Brown and White (2005), prior to calculating $\log d$. Values of $\log d$ for the two replications of each condition were averaged, and subjected to repeated measures analysis of variance. A preliminary analysis was also conducted, in which the proportion of correct red choices following red samples and the proportion of correct green choices following green sample was calculated at each delay in order to assess the biasing effect of the different rewards for the different choices on differential outcome trials, and to provide a general indication of overall performance accuracy.

\section{Results and discussion}

Figure 1 shows the proportion of choices of red following red samples, and the proportion of green choices following green samples (proportion of correct red or green choices respectively) as a function of delay, averaged across pigeons, for the SDS and DSD conditions. Figure 1 also illustrates the overall level of accuracy which was generally high, and more importantly, the biasing effect of the differential outcomes. The biasing effect at a given delay is indicated by a difference between the proportion of correct choices of red (which produced 1.5-s rewards on different outcome trials) and the proportion of correct choices of green (which produced 4.5-s rewards on different outcome trials).

A repeated measures analysis of variance confirmed a significant effect of delay, $F(2,6)=14.78, p=.005, M S E=$ $0.008, \eta_{\mathrm{p}}{ }^{2}=.83$, and a three-way interaction between SDS versus DSD condition, delay, and correct red versus green

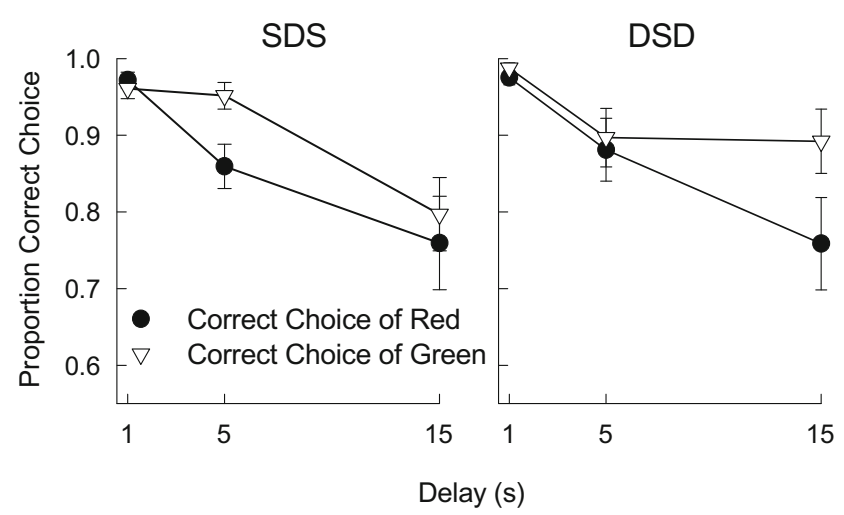

Fig. 1 Proportion correct choice of red following red samples (filled circles) and proportion correct choice of green following green samples (unfilled triangles) averaged across pigeons in conditions with Same, Different, and Same outcomes respectively at 0.5-, 5-, and 15-s delays (SDS) or with Different, Same, and Different outcomes respectively at $0.5,5$, and 15-s delays (DSD) in Experiment 1. On Same outcome trials, correct red and correct green choices both produced 3-s access to wheat. On Different outcome trials, correct red choices produced 1.5-s access to wheat and correct green choices produced 4.5-s access to wheat. Error bars represent the standard error of the mean 
response types, $F(2,6)=12.95, p=.007, M S E=0.001, \eta_{\mathrm{p}}{ }^{2}=$ .81. There were no other statistically significant main effects or interactions. In the SDS condition, in which differential outcomes were arranged at the 5-s delay, the proportion of correct choices of green were significantly higher than the proportion of correct choices of red at $5 \mathrm{~s}$, reflecting the biasing effect of the larger reward for green choices (Neuman Keuls $p=.009$ ). The proportion correct red and green choices at 0.5 -s and 15 -s delays (at which same outcomes were arranged in the SDS condition) were not significantly different. In the DSD condition, in which differential outcomes were arranged at 0.5 -s and 15 -s delays, the proportion of correct green choices was greater than the proportion of correct red choices at the 15-s delay (Neuman Keuls $p=.002$ ), but not significantly so at $0.5 \mathrm{~s}$, consistent with the general observation that the biasing effect of differential outcomes tends to be large at longer delays and minimal at short delays where discriminability is high (White \& Wixted, 1999, 2010). In sum, the statistically significant three-way interaction confirms the different biasing effect of the differential outcomes at the different delays in the SDS and DSD conditions.

In the following analysis, we assessed accuracy of delayed matching using the discriminability measure, $\log d$ (Eq. 1), because proportion correct is affected by response bias, and by ceiling effects where accuracy is very high, as at the 0.5 -s delay. Forgetting functions therefore plotted discriminability as a function of delay (White, 2013). Figure 2 shows forgetting functions for each pigeon, and for the mean across pigeons, for SDS and DSD conditions in Experiment 1. Instances of a DOE at specific delays occurred at 0.5 -s and 15$\mathrm{s}$ delays when discriminability in the DSD condition (unfilled triangles) was higher than in the SDS condition (filled circles). This occurred for each pigeon at $0.5 \mathrm{~s}$, and for pigeons $\mathrm{H} 1, \mathrm{H} 2$ (to a small extent), and $\mathrm{H} 3$ at $15 \mathrm{~s}$. Additionally, a DOE at $5 \mathrm{~s}$, when discriminability was higher in the SDS condition than in the DSD condition, occurred for pigeons H1, H2, and H3.

A delay-specific DOE would be evident at each of the delays if a full "cross-over" interaction between condition and delay occurred. Pigeons $\mathrm{H} 1$ and $\mathrm{H} 3$ showed this full interaction in which discriminability at .5-s and 15 -s delays was higher in the DSD condition than in the SDS condition, and discriminability at the 5-s delay was higher in the SDS condition than the DSD condition. A similar interaction occurred for Pigeon H2, although a DOE was very small at $15 \mathrm{~s}$ for $\mathrm{H} 2$. Pigeon $\mathrm{H} 4$ did not exhibit the full cross-over interaction. The full cross-over interaction can be seen in the mean data, but the interaction between condition and delay in a repeated measures analysis of variance was not statistically significant, $F(2,6)=$ $1.99, p=.22$. Of the 12 possible instances across the four pigeons and three delays at which a DOE could occur, however, there were ten cases when accuracy at a delay followed by differential outcomes was higher than at the same delay with same outcomes for correct choices (Sign test $p=.02$ ). We conclude

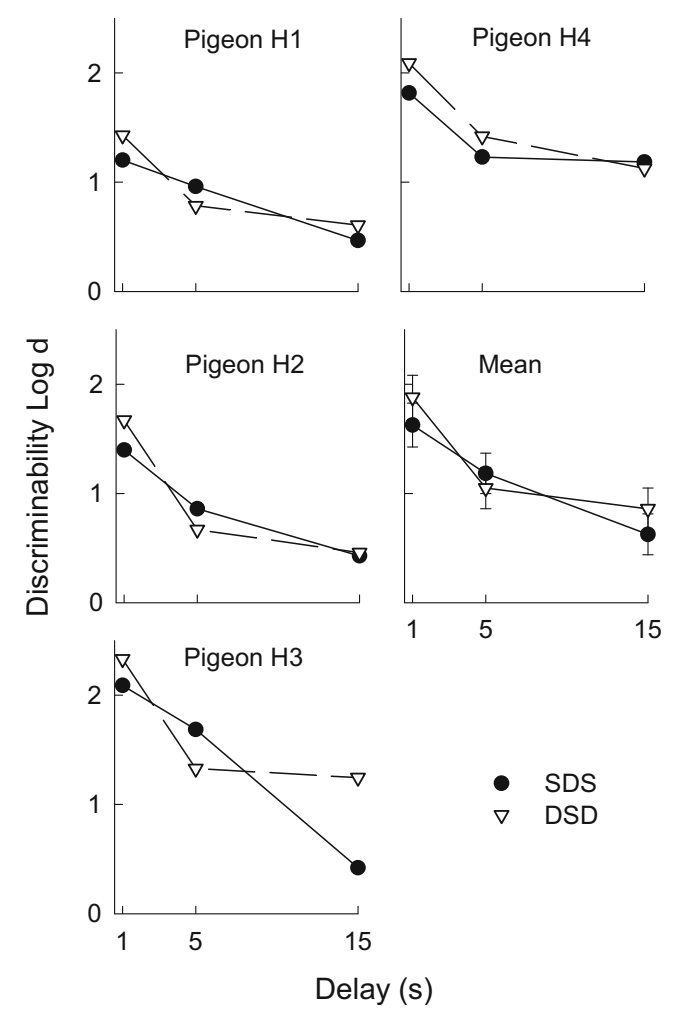

Fig. 2 Discriminability $(\log d)$ as a function of delay $(0.5,5$, and $15 \mathrm{~s})$ for each pigeon, and for the mean across pigeons, for the Same-DifferentSame (SDS; filled circles) and the Different-Same-Different (DSD; unfilled triangles) conditions in Experiment 1. Error bars in the mean graph represent the standard error of the mean

that for three of the four pigeons, there was sufficient evidence to suggest the occurrence of delay-specific DOEs, with clear evidence in the case of pigeons $\mathrm{H} 1$ and $\mathrm{H} 3$. The absence of the effect in pigeon $\mathrm{H} 4$, however, suggests that the evidence may be limited.

One problem with Experiment 1 was that the DSD condition had two delays with different outcomes whereas the SDS condition had only one. This may have resulted in overall higher discriminability in the DSD than in the SDS condition, although the main effect of condition in the repeated measures analysis of variance was not statistically significant, $F(1,3)=$ $6.88, p=.08$. Additionally, accuracy at the same-outcome delay in DSD may have been higher as a result of possible generalization of performance from the proximal differentialoutcome delays. In DSD, there were two delays from which accuracy could generalize ( 0.5 and $15 \mathrm{~s})$, so improved accuracy at both these delays could improve accuracy at the intermediate delay of $5 \mathrm{~s}$. The effect of generalization from two delays with differential outcomes surrounding an intermediate delay with same outcomes would likely be greater than the generalization from a single differential-outcome intermediate delay to two same-outcome surrounding intervals. Thus, it is likely that the DSD conditions produced enhanced performance at the intermediate delay due to generalization of 
discriminability, similar to the generalization of performance reported by Sargisson and White (2001). In order to take account of the imbalance of outcome type between the two conditions in Experiment 1, Experiment 2 was designed to balance the number of same and differential-outcome delays across conditions.

\section{Experiment 2}

In Experiment 2, four pigeons were trained with delays of 0, 3, and $12 \mathrm{~s}$, mixed within sessions. In the SDS condition, correct responses at 0 -s and 12-s delays were followed by the same outcomes whereas at the 3-s delay, correct responses produced differential outcomes. In the SSD condition, correct responses after the 0 - and 3-s delays were followed by same outcomes and correct responses at the 12-s delay produced differential outcomes. Thus each condition included just one delay of three in which outcomes for correct choices were differential. As in Experiment 1, the random order of delays meant that trials in which outcomes might be the same or differential could not be predicted, and expectancies of differential outcomes could be unlikely, especially considering that twothirds of trials ended in same outcomes. Two conditions were included in which same outcomes followed correct choices at each of the three delays, in order to allow an across-condition comparison of the effect of same versus differential outcomes. The procedure was generally similar to that in Experiment 1, but with a different set of delays and different reinforcer durations.

\section{Method}

Subjects Four homing pigeons (Columba livia) different from those in Experiment 1, T1 to T4, were maintained at above $85 \%$ of their free-feeding weights by supplementary feeding of mixed grain. They were individually housed with free access to water and grit in a colony room with a 12-h:12-h light:dark cycle supplemented by natural light. The pigeons had had a month's prior experience in DMTS with red and green samples and short delays, following initial keypeck training.

Apparatus Four Med Associates operant chambers, $29.5 \mathrm{~cm}$ wide, $24.5 \mathrm{~cm}$ deep, and $29.5 \mathrm{~cm}$ high above a wire grid floor were used. Three circular response keys on one of the chamber walls were $6 \mathrm{~cm}$ apart (center to center), $21 \mathrm{~cm}$ from the wire floor, and $3.5 \mathrm{~cm}$ from each side of the wall. Each key was $2.1 \mathrm{~cm}$ in diameter and could be illuminated green or red. Positioned $12.5 \mathrm{~cm}$ below the center key and $4 \mathrm{~cm}$ above the wire floor was an opening $4.5 \mathrm{~cm}$ high, $6 \mathrm{~cm}$ wide, and $4 \mathrm{~cm}$ deep containing a hopper which could be raised to provide the pigeon with access to wheat. Entry of the pigeon's head into the hopper opening was detected by a photocell beam. A house-light was mounted on a side wall. Ventilation fans masked extraneous noise. The experiments were controlled and recorded by a computer located in an adjacent room running MedPC, and MedPC interfacing equipment and software (Version IV).

Procedure Each daily session of 96 trials began when the center key was lit either red or green. After five pecks to the center key, a delay was programmed, after which one side key was lit red and the other green. A single peck to the comparison key which matched the color of the sample resulted in reinforcement, followed by a 12 -s ITI. Incorrect choices of comparison stimuli led directly to the ITI. Trial-by-trial selection of sample key colors and left and right key colors for comparison choices was achieved using lists, so that each sample color was selected equally often, and was followed equally often by red and green comparison choices on the left and right keys. A single dummy trial, and four random trials, preceded the 96 trials, but the data from these five trials were excluded from the analysis.

On same-outcome (S) trials, reinforcement for correct comparison choices was 2.5-s access to wheat both when the sample was red and when it was green. On differential-outcome (D) trials, 4.5-s access to wheat served as the reinforcer for correct red choices, and 0.5-s access for correct green choices. The reinforcer duration was timed from the moment that the pigeon placed its head in the hopper opening.

For preliminary training in the first 12 sessions, the withinsession delays were $0,1,3,6$, or $12 \mathrm{~s}$, with same outcomes following each delay. For the next 13 sessions of training in the first of six conditions, delays of 0,3 , and $12 \mathrm{~s}$ were used with same outcomes following each delay. Subsequent conditions were each conducted for 20 sessions. Table 1 shows the six conditions in Experiment 2 and whether the outcome was the same (S) or different (D) after each delay in each condition. Data analysis was conducted in the same way as in Experiment 1, based on correct and error responses at each delay summed over the last five sessions of each condition.

Table 1 Order of conditions in Experiment 2, showing same (S) or different (D) outcome contingencies for each of the three delays

\begin{tabular}{llll}
\hline & \multicolumn{2}{l}{ Delays (s) } & \\
\cline { 2 - 4 } Condition & 0 & 3 & 12 \\
\hline 1 & $\mathrm{~S}$ & $\mathrm{~S}$ & $\mathrm{~S}$ \\
2 & $\mathrm{~S}$ & $\mathrm{D}$ & $\mathrm{S}$ \\
3 & $\mathrm{~S}$ & $\mathrm{~S}$ & $\mathrm{D}$ \\
4 & $\mathrm{~S}$ & $\mathrm{~S}$ & $\mathrm{~S}$ \\
5 & $\mathrm{~S}$ & $\mathrm{~S}$ & $\mathrm{D}$ \\
6 & $\mathrm{~S}$ & $\mathrm{D}$ & $\mathrm{S}$ \\
\hline
\end{tabular}


Results and discussion

Figure 3 shows the mean proportion of correct red or green choices as a function of delay for the SDS and SSD conditions. A repeated measures analysis of variance showed a significant effect of delay, $F(2,6)=13.94, p=.006, M S E=$ $0.06, \eta_{\mathrm{p}}^{2}=.82$, a two-way interaction between SDS versus SSD condition, and correct red versus green response types, $F(1,3)=11.09, p=.045, M S E=0.003, \eta_{\mathrm{p}}^{2}=.79$, and a threeway interaction between condition, delay, and correct red versus green response types, $F(2,6)=18.66, p=.003, M S E=$ $0.003, \eta_{\mathrm{p}}^{2}=.86$. There were no other statistically significant main effects or interactions. In the SDS condition, in which differential outcomes were arranged at the 3-s delay, proportion of correct choices of red (leading to the 4.5 -s reward) were higher than proportion of correct choices of green (leading to the 0.5 -s reward), but the difference was not statistically significant (Neuman Keuls $p>.05$ ), although it occurred for three of four pigeons. Proportion correct red and green choices at 0 -s and 12-s delays (at which same outcomes were arranged in the SDS condition) were not significantly different. In the SSD condition, in which differential outcomes were arranged at the 12-s delay, the proportion of correct red choices was greater than the proportion of correct green choices at the 12-s delay (Neuman Keuls $p=.003$ ), reflecting the biasing effect of the larger reward for red choices. Proportion correct red and green choices were not significantly different at the 0 -s and 3-s delays, at which same outcomes were arranged in the SSD condition.

$\log d$ values were averaged for the two SDS conditions in which a differential outcome was scheduled only after the 3-s delay (Conditions 2 and 6), and for the two SSD conditions in

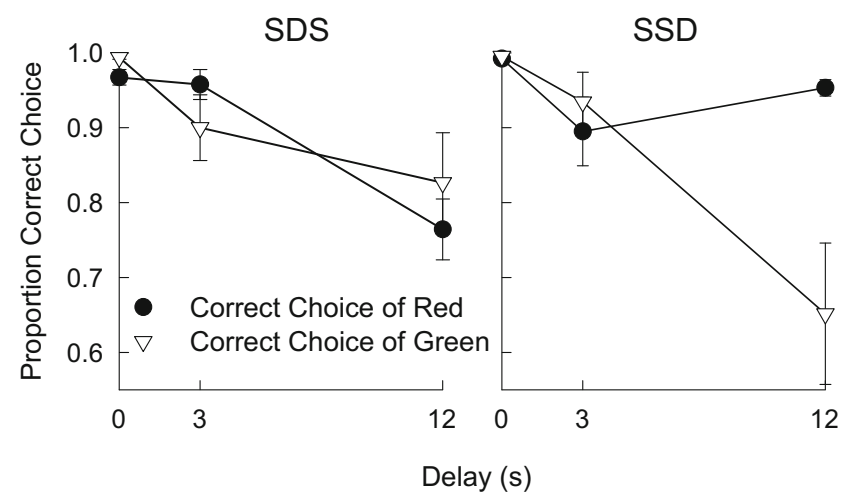

Fig. 3 Proportion correct choice of red following red samples (filled circles) and proportion correct choice of green following green samples (unfilled triangles) averaged across pigeons in conditions with Same, Different, and Same outcomes respectively at 0-, 3-, and 12-s delays (SDS) or with Same, Same, and Different outcomes respectively at 0-, 3-, and 12-s delays (SSD) in Experiment 2. On Same outcome trials, correct red and correct green choices both produced 2.5-s access to wheat. On Different outcome trials, correct red choices produced 4.5-s access to wheat and correct green choices produced 0.5 -s access to wheat. Error bars represent the standard error of the mean which the differential outcome was arranged only after the 12s delay (Conditions 3 and 5). Figure 4 shows discriminability $(\log d)$ in the SDS and SSD conditions for each pigeon and for the mean across pigeons. Figure 4 shows that after the 12-s delay, discriminability was higher in the SSD condition when differential outcomes were arranged than when same outcomes were arranged in the SDS condition. In contrast, at the 3-s delay, discriminability in the SDS condition was higher than in the SSD condition for pigeons T1 and T4, although discriminability at $3 \mathrm{~s}$ did not differ between the two conditions for pigeons T2 and T3. A repeated measures analysis of variance was conducted on discriminability measures at $3 \mathrm{~s}$ and $12 \mathrm{~s}$ (at $0 \mathrm{~s}$, same outcomes were arranged in both SDS and SDD conditions). The interaction between condition and delay evident in the mean data in Fig. 4 was statistically significant, $F(1,3)=14.23, p=.03, M S E=0.01, \eta_{\mathrm{p}}{ }^{2}=.83$, consistent with a delay-specific DOE.

Two conditions were conducted in which outcomes were the same at all three delays (SSS). Discriminability at $0 \mathrm{~s}$ was not expected to differ between SSS, SDS, and SSD conditions (despite the differences evident in Fig. 3 for SDS and SSD conditions), because same outcomes followed correct choices at the 0 -s delay in all conditions. Indeed, it did not, according to a one-way repeated measures analysis of variance on

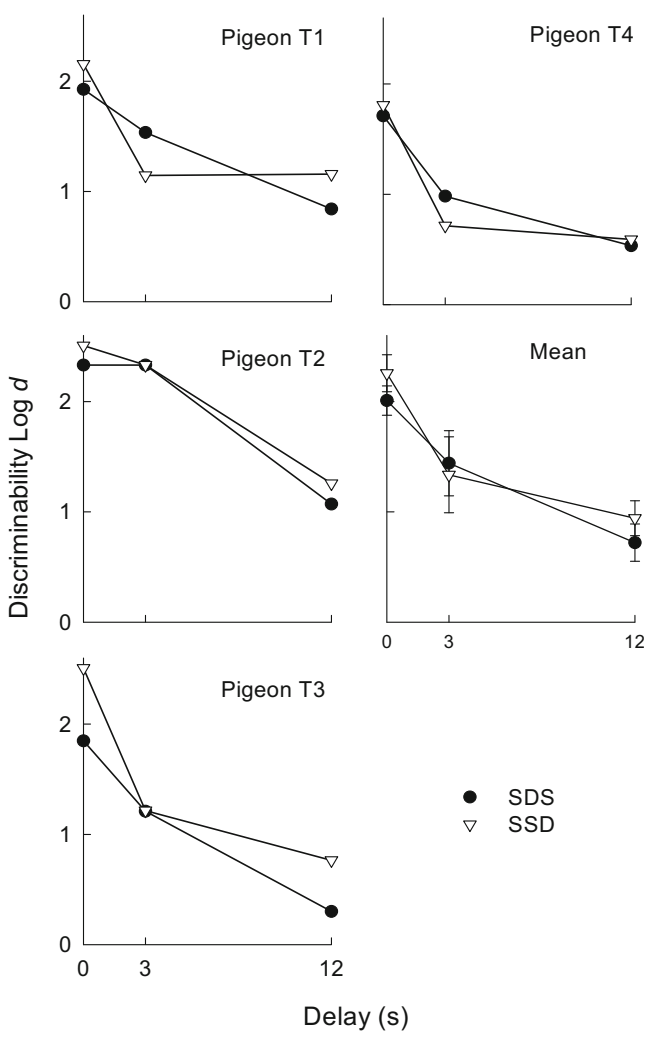

Fig. 4 Discriminability $(\log d)$ as a function of delay $(0,3$, and $12 \mathrm{~s})$ for each pigeon, and for the mean across pigeons, for the Same-DifferentSame (SDS; filled circles) and the Same-Same-Different (SSD; unfilled triangles) conditions in Experiment 2. Error bars in the mean graph represent the standard error of the mean 
discriminability measures at $0 \mathrm{~s}$ in the three conditions, in which the effect of condition was not statistically significant, $F(2,6)=3.74, p=.09$. Although $\log d$ at $0 \mathrm{~s}$ was higher in the SSD condition than in the SDS condition for each pigeon, despite the outcomes being the same at $0 \mathrm{~s}$ (Fig. 4), this difference was not statistically significant (Neuman Keuls $p=.09$ ).

Mean discriminability at 3-s and 12-s delays in the SSS conditions was used to assess the extent of a delay-specific DOE on a between-conditions basis. Figure 5 shows discriminability for each pigeon, separately for the 3 -s (top panel) and the 12-s (bottom panel) delays. In the top panel, when outcomes were the same following all delays (black bars), discriminability at $3 \mathrm{~s}$ was lower for each pigeon than in the SDS condition when the outcome for just the 3-s delay was different. For 12-s delays (bottom panel), discriminability was higher for each pigeon when correct responses were differentially reinforced in the SSD condition than when correct responses produced the same outcomes in the SSS condition. A repeated measures analysis of variance on the data in Fig. 5 showed significant main effects of delay, $F(1,3)=14.02$, $p=.03, M S E=.10, \eta_{\mathrm{p}}{ }^{2}=.82$, and, more importantly, of same versus different outcome condition, $F(1,3)=34.84, p=.01$, $M S E=0.03, \eta_{\mathrm{p}}{ }^{2}=.92$, with no interaction between them, $F<1$. The significant difference between same and differential outcome conditions at each delay in Fig. 5 provides further evidence for a delay-specific DOE.

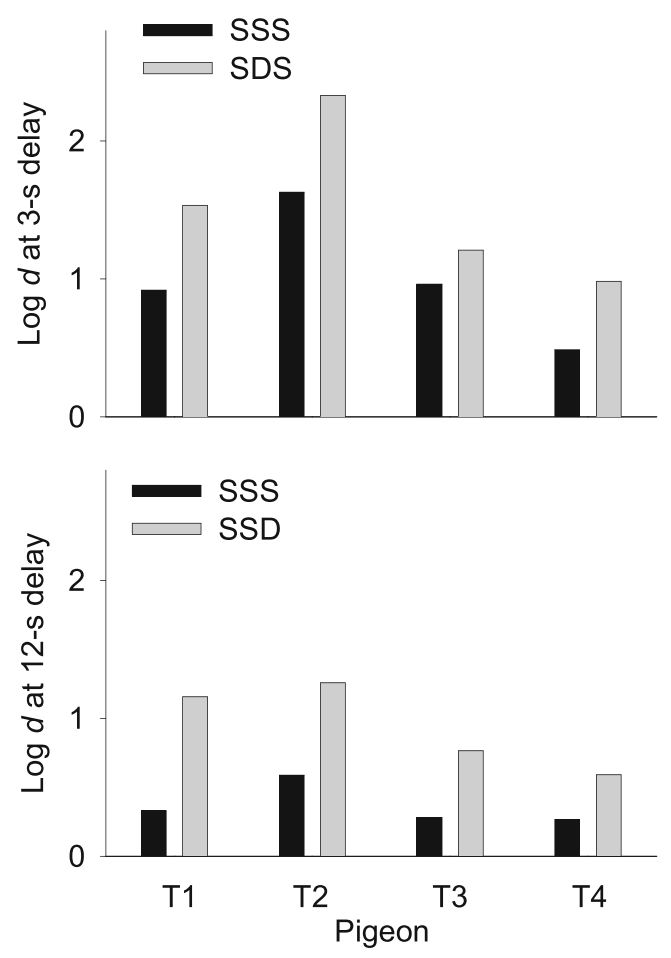

Fig. 5 Discriminability $(\log d)$ at 3-s delays in Same-Same-Same (SSS) and the Same-Same-Different (SSD; top panel) and at 12-s delays in SSS and SSD conditions (bottom panel) for each pigeon in Experiment 2
This latter conclusion is compromised, however, by the result that discriminability with same outcomes at $3 \mathrm{~s}$ and $12 \mathrm{~s}$ in the SSD and SDS conditions, respectively, was higher than discriminability at the corresponding delays in the SSS condition. In other words, inclusion of a differential outcome at one delay generalized its enhancing effect to delays at which outcomes were the same. The interaction between delay and conditions in a repeated measures analysis of variance on $\log d$ for all three conditions and $3 \mathrm{~s}$ and $12 \mathrm{~s}$ delays was significant, $F(2,6)=8.25, p=.02, M S E=0.01, \eta_{\mathrm{p}}{ }^{2}=.73$, as were differences in discriminability between SSS and SSD or SDS conditions at $3 \mathrm{~s}$ and $12 \mathrm{~s}$ delays respectively (Neuman Keuls $p=.001 ; p=.005$ ), despite the outcomes being the same in both cases. Nonetheless, compared to discriminability at 3-s and 12-s delays in the SSS condition, the absolute differences in mean discriminability at 3-s and 12-s delays at which differential outcomes were arranged in SDS and SSD conditions were 0.52 and 0.58 , whereas the corresponding differences of 0.32 and 0.35 in comparisons involving same outcomes were significantly smaller, $F(1,3)=14.23, p=.03, M S E=0.01, \eta_{\mathrm{p}}{ }^{2}$ $=.83$, and did not depend on delay, $F<1$. That is, the generalization effect was smaller than the direct effect of differential outcomes at a specific delay, although it weakens the evidence for a delay-specific DOE.

\section{Experiment 3}

The aim of Experiment 3 was to confirm the occurrence of the DOE for the general procedural conditions of Experiments 1 and 2, but using a more standard between-conditions comparison. Discriminability at three delays all with same outcomes in the SSS was compared to discriminability at three delays all with differential outcomes in the DDD condition.

Method

The four pigeons from Experiment 2 continued directly to Experiment 3 . The apparatus and procedure were exactly the same as in Experiment 2, except that same outcomes were arranged at each of the 0-, 3-, and 12-s delays in the SSS condition, and differential outcomes were arranged at each of the 0-, 3-, and 12-s delays in the DDD condition. As in Experiment 2, on same-outcome (S) trials, correct choices of both red and green produced 2.5 -s access to wheat, and on differential-outcome (D) trials, 4.5 -s access to wheat followed correct red choices, and 0.5-s access for correct green choices.

Each condition was replicated, and each was conducted for 20 sessions, in the order SSS, DDD, SSS, and DDD.

Data analysis was the same as in Experiments 1 and 2, with the exception that discriminability was calculated for each of four successive blocks of five sessions in each condition, and a preliminary analysis of proportion of correct red or green 
choices was not included. In addition, a measure of response bias was calculated. This measure, $\log b$, was similar in structure to $\log d$, but instead of being based on ratios of correct to error responses, it was based on ratios of choices of red to green comparisons. Thus $\log b$ measured the extent to which choice between comparison stimuli was biased towards choosing either color. Positive values of $\log b$ would indicate a bias towards choosing red. The same correction was applied in the calculation of $\log b$ as recommended by Brown and White (2005). The bias measure (in which errors are choices of red and green following green and red samples respectively) was:

$\log b=0.5 \cdot \log \left(\left(\frac{c_{\text {red }}}{c_{\text {green }}}\right)\left(\frac{e_{\text {green }}}{e_{\text {red }}}\right)\right)$

Results and discussion

Figure 6 shows $\log d$ (top panels) and $\log b$ (bottom panels) plotted as a function of delay across four successive blocks of five sessions each for SSS conditions (filled circles) and DDD conditions (unfilled triangles). The $\log d$ and $\log b$ measures in Fig. 6 are the averages across pigeons of means for each block of the two replications of SSS and DDD conditions. The patterns of data for individual pigeons were similar, as indicated by the small standard errors of the mean ( 40 panels would be required to show individual data).

\section{Bias}

Log $b$ was calculated using Eq. 2 in order to confirm that the pigeons equally preferred red and green comparison stimuli across delays in the SSS conditions, in which outcomes of red and green choices were the same. Additionally, $\log b$ was calculated to verify that the differential outcomes at each delay in the DDD conditions generated a preference for the choice followed by the larger reinforcer. Figure 6 (lower panels) shows that both assumptions were correct $-\log b$ remained constant across delays and blocks at close to indifference (log $b=0)$ in SSS conditions, and at $\log b=0.83$ in DDD conditions, that is a ratio of about 6.8/1 in favor of choosing red, the comparison associated with the larger reinforcer. It might be noted that the ratio of red/green reinforcer durations was $9 / 1$, but undermatching of response ratios to ratios of reinforcer durations is not unusual (Davison, 1988). A repeated measures analysis of variance confirmed the statistically significant main effect of SSS versus DDD condition on $\log b$, $F(1,3)=58.69, p=.005, M S E=0.33, \eta_{\mathrm{p}}{ }^{2}=.95$. The effects of delay and block were not significant and there was no significant interaction between condition, block, and delay. We conclude, therefore, that in the SSS conditions, the same outcomes led to equal preference at each delay, and in the DDD conditions, the differential outcomes biased choice towards the larger outcome to the same extent across delays and blocks of sessions.

\section{Discriminability}

Figure 6 (top panel) shows that, in general, $\log d$ decreased with increasing delay, $F(2,6)=23.48, p=.001, M S E=0.25$, $\eta_{\mathrm{p}}{ }^{2}=.89$. The overall effect of SSS versus DDD condition was not statistically significant, $F<1$, but there was a significant interaction between SSS versus DDD condition and delay, $F(2,6)=9.34, p=.01, M S E=0.13, \eta_{\mathrm{p}}^{2}=.76$. This interaction, in which the rate of forgetting in the same outcomes condition is greater than that in the differential outcomes condition, is typical of the DOE (Jones \& White, 1994; White \& Millar, 2014).

The only other statistically significant effect in the analysis of variance on $\log d$ measures shown in the top panel of Fig. 6 was the three-way interaction between SSS versus DDD conditions, delay, and blocks of sessions, $F(6,18)=4.08, p=$

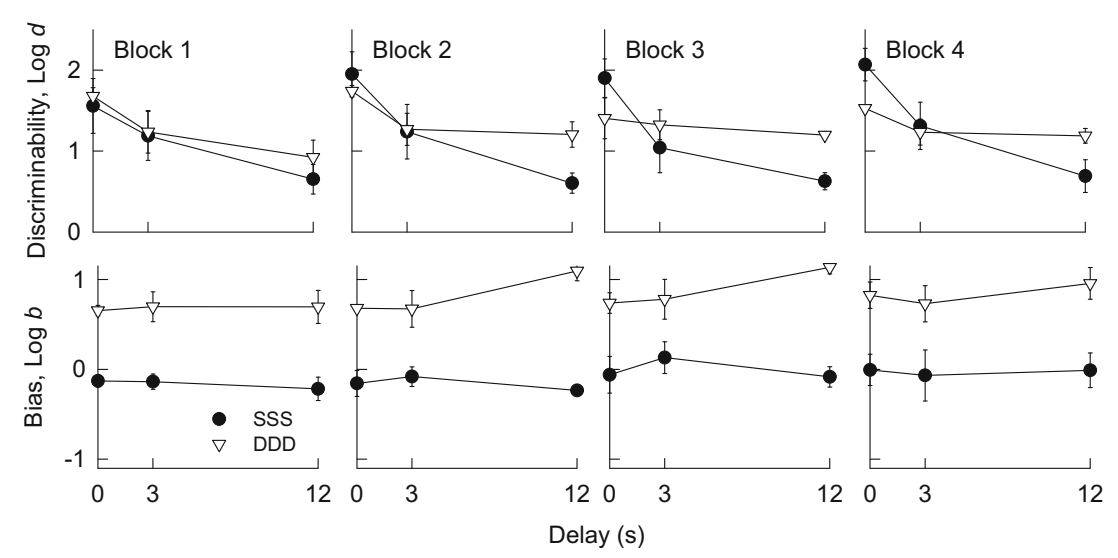

Fig. 6 Mean discriminability $(\log d$ ) (top panels) and mean response bias $(\log b)$ (bottom panels) as a function of delay for successive blocks of five sessions of Same-Same-Same (SSS; filled circles) and Different-
Different-Different (DDD; unfilled triangles) conditions in Experiment 3. Error bars represent the standard error of the mean 
$.009, M S E=0.51, \eta_{\mathrm{p}}{ }^{2}=.58$. In the first block of five sessions, functions for SSS and DDD conditions overlapped, and did not diverge to show the typical DOE at the long delay until the second block of five sessions. By then there was a significant difference between $\log d$ for the two conditions at the 12-s delay (Newman-Keuls $p=.003$ ). The DOE at $12 \mathrm{~s}$ persisted in the third $(p=.003)$ and fourth $(p=.003)$ blocks of sessions. In the third and fourth blocks of five sessions, however, while the DOE at $12 \mathrm{~s}$ had persisted, a reverse DOE appeared at the $0-\mathrm{s}$ delay in which $\log d$ in SSS was higher than for DDD conditions ( $p=.007$ in the third block, $p=.004$ in the fourth block). This reversal seems to be the result of high levels of accuracy at $0 \mathrm{~s}$ in SSS conditions, combined with the strong response bias in DDD conditions which inevitably suppresses discriminability by creating errors.

In conclusion, the results of Experiment 3 confirm the occurrence of the DOE at longer (12-s) delays in DMTS, and suggest that at shorter delays ( $0 \mathrm{~s}$ or $3 \mathrm{~s}$ ), the DOE may not necessarily occur, at least for the present procedures. Additionally, the unusual reversal of the DOE at $0 \mathrm{~s}$ in the third and fourth blocks of five sessions in the present conditions underlines the fascinating paradox of the DOE - in the face of response bias which creates errors and reduces discriminability, the DOE is manifest as an enhancement in discriminability. Perhaps the DOE is likely to be seen when overall discriminability levels are relatively low, such as those at long delays or retention intervals.

\section{General discussion}

In the present experiments, we arranged differential outcomes for correct matching responses at some delays but not at others, with three delays mixed within sessions on an unpredictable basis. Several individual pigeons showed delayspecific DOEs, in that discriminability was enhanced at delays with differential outcomes, and not at delays with the same outcomes. The occurrence of delay-specific remembering for the DOE adds to other evidence for delay-specific remembering (e.g., White, 2001; White \& Brown, 2011a, b). The demonstration of a delay-specific DOE in Experiments 1 and 2 should be qualified, however, by noting that the effects did not occur in all possible cases for all pigeons, and when they did occur, the effects tended to be small, and smaller in magnitude than the DOE found in Experiment 3, in which the same or differential outcomes were arranged at all three delays within each session. Additionally, the results of Experiment 2 suggested that the effects of differential outcomes at one delay might generalize to other delays, consistent with other evidence for the generalization of performance from one delay to adjacent delays (Sargisson \& White, 2001), thus limiting the delay-specificity of the DOE. Nonetheless, there seems to be sufficient evidence from the present experiments to conclude that a delay-specific DOE is possible.

The occurrence of a delay-specific DOE seems inconsistent with the main theoretical account for the DOE, that the DOE results from an outcome expectancy generated at the time of sample presentation and carried through the retention interval to amplify stimulus control over choice responding. Because the pigeon could not predict whether a trial would end in same or differential outcomes, it seems unlikely that an appropriate expectancy would be formed at the time of sample presentation. To account for the possibility of delay-specific effects of differential outcomes, and perhaps also their generalization to proximal delays, an expectancy could be assumed to change over the course of the retention interval. For example, in the SSD condition, differential sample-outcome expectancies would be conditional on time approaching the longest delay. Inclusion of time spent in the retention interval as an additional discriminative cue is analogous to using an explicit discriminative stimulus to cue differential-outcome trials versus same-outcome trials mixed within session, as Jones and White (1994) did. It is therefore plausible that time in the retention interval could cue the outcomes specific to a delay, as well as outcome expectancies. In other words, if a differential-outcome expectancy mediated performance, it would be generated close to the time of the delay at which the differential outcomes were programmed, and not at the time of sample presentation.

A delay-specific DOE is consistent with the view that remembering, at least in DMTS, is specific to the time at which remembering is required (White, 2001, 2002). In other words, the choice between comparison stimuli is influenced by a compound (or conditional discrimination) which includes the comparison stimuli, the earlier-presented sample, and also the current delay. Differential outcomes therefore enhance the discrimination made at the time of the choice between comparison stimuli.

Consistent with this view, DeMarse and Urcuioli (1993) argued that associations between differential outcomes and comparison stimuli were sufficient to enhance matching accuracy in a conditional discrimination. They pointed to the observation made by Honig, Matheson, and Dodd (1984) that when samples in a conditional discrimination are correlated with different outcomes, so too are the comparison stimuli. Enhanced accuracy with differential outcomes could therefore result from the association of the different outcomes with the comparison stimuli, not the sample stimuli (also see Urcuioli, 2005).

DeMarse and Urcuioli (1993) arranged three conditions for different groups of pigeons. Their measure was rate of learning a conditional discrimination. In all three groups of their Experiment 1, choices of a vertical line or red were reinforced following one sample and choices of a horizontal line or green were reinforced following the other sample. In one control group, reinforcers were food delivered with a probability of 
1.0. For the second control group, reinforcer probability was 0.5. After 14 sessions of training, accuracy in these one-tomany matching procedures with same outcomes improved. Accuracy for the first control group reached nearly $60 \%$, and for the second control group, reached about $68 \%$. For the experimental group, reinforcers were food following choices of vertical and green, and feeder-light flashes following choices of horizontal and red. These differential outcomes followed the two samples equally often and thus were not correlated with the sample stimuli. In this condition, accuracy climbed to about $90 \%$ after 14 sessions. DeMarse and Urcuioli argued that a DOE therefore does not require a differential association between outcomes and samples.

In a second experiment, DeMarse and Urcuioli (1993) repeated their first experiment, but with a reinforcer probability of 0.6 for each correct choice in a same-outcomes control group, and 1.0 versus 0.2 probabilities of food as differential outcomes in the experimental group. As in their Experiment 1, the differential outcomes of choice responses were not correlated with sample stimuli. After 40 sessions, the experimental group achieved $90 \%$ whereas the control group achieved $82 \%$ accuracy. It therefore seems that an association between comparisons and differential outcomes for correct choice responses, independently of sample stimuli, are sufficient to create a DOE. This result, and the results of other studies in which samples were not correlated with outcomes (e.g., DeMarse \& Urcuioli, 2005), are consistent with the possibility that differential-outcome expectancies can arise from serial sample-comparison compounds, as suggested by Urcuioli, DeMarse, and Lionello (1998).

A delay-specific DOE, as demonstrated here, is consistent with the notion of a serial sample-comparison compound, given that time in the retention interval might also be incorporated in the compound. Potentially, the discrimination made at the time of the choice between comparison stimuli benefits from the differential outcomes produced by correct choices, and that benefit depends on the delay and can be short-lived. If an outcome expectancy is generated by the compound, however, it would need to occur close to the time of presentation of the comparison stimuli, because the delays and associated outcomes in the present experiments were mixed randomly within session. Whether the delay-specific DOE is a result of an enhanced discrimination by delay-specific outcome expectancies may, however, be a difficult notion to test experimentally.

\section{References}

Brodigan, D. L., \& Peterson, G. B. (1976). Two-choice conditional discrimination performance of pigeons as a function of reward expectancy, prechoice delay, and domesticity. Animal Learning \& Behavior, 4, 121-124.
Brown, G. S., \& White, K. G. (2005). The optimal correction for estimating extreme discriminability. Behavior Research Methods, 37, 436-449.

Davison, M. (1988). Concurrent schedules: Interaction of reinforcer frequency and reinforcer duration. Journal of the Experimental Analysis of Behavior, 49, 339-349.

Davison, M. C., \& Tustin, R. D. (1978). The relation between the generalized matching law and signal detection theory. Journal of the Experimental Analysis of Behavior, 51, 291-315.

De Long, R. E., \& Wasserman, E. A. (1981). Effects of differential reinforcement expectancies on successive matching-to-sample performance in pigeons. Journal of Experimental Psychology: Animal Behavior Processes, 7, 394-412.

DeMarse, T. B., \& Urcuioli, P. J. (1993). Enhancement of matching acquisition by differential comparison-outcome associations. Journal of Experimental Psychology: Animal Behavior Processes, 19, 317-326.

DeMarse, T. B., \& Urcuioli, P. J. (2005). Control of matching by differential outcomes expectancies in the absence if differential sampleoutcome associations: A serial compound view. Journal of Experimental Psychology: Animal Behavior Processes, 31, 449-466.

Estevez, A. F., Overmier, B., \& Fuentes, L. J. (2003). Differential outcomes effect in children: Demonstration and mechanisms. Learning and Motivation, 34, 148-167.

Friedrich, A. M., \& Zentall, T. R. (2011). A differential-outcome effect in pigeons using spatial hedonically nondifferential outcomes. Learning \& Behavior, 39, 68-78.

Holden, J. M., \& Overmier, J. B. (2014). Performance under differential outcomes: Contributions of reward-specific expectancies. Learning and Motivation, 45, 1-14.

Honig, W. K., Matheson, W. R., \& Dodd, P. W. D. (1984). Outcome expectancies as mediators for discriminative responding. Canadian Journal of Psychology, 38, 196-217.

Jones, B. M., \& White, K. G. (1994). An investigation of the differentialoutcomes effect within sessions. Journal of the Experimental Analysis of Behavior, 61, 389-406.

Legge, E. L. G., \& Spetch, M. L. (2009). The differential outcomes effect (DOE) in spatial localization: An investigation with adults. Learning and Motivation, 40, 313-328.

McDonald, S. E., \& Grant, D. S. (1987). Effects of signalling retention interval length on delayed matching-to-sample in pigeons. Journal of Experimental Psychology: Animal Behavior Processes, 13, 116-125.

Miyashita, Y., Nakajima, S., \& Imada, H. (2000). Differential outcome effect in the horse. Journal of the Experimental Analysis of Behavior, $74,245-253$.

Nakagawa, S., Etheridge, R. J. M., Foster, T. M., Sumpter, C. E., \& Temple, W. (2004). The effects of changes in consequences on hens' performance in delayed-matching-to-sample tasks. Behavioural Processes, 67, 441-451.

Nevin, J. A., \& Grosch, J. (1990). Effects of signalled reinforcer magnitude on delayed matching-to-sample performance. Journal of Experimental Psychology: Animal Behavior Processes, 16, 298-305.

Nevin, J. A., Ward, R. D., Jimenez-Gomez, C., Odum, A. L., \& Shahan, T. A. (2009). Differential outcomes enhance accuracy of delayed matching to sample but not resistance to change. Journal of Experimental Psychology: Animal Behavior Processes, 35, 74-91.

Overmier, B., Bull, J. A., III, \& Trapold, M. A. (1971). Discriminative cue properties of different fears and their role in response selection in dogs. Journal of Comparative and Physiological Psychology, 76, 478-482.

Peterson, G. B., Linwick, D., \& Overmier, J. B. (1987). On the comparative efficacy of memories and expectancies as cues for choice behavior in pigeons. Learning and Motivation, 18, 1-20. 
Santi, A., \& Roberts, W. A. (1985). Prospective representation: the effects of varied mapping of sample stimuli to comparison stimuli and differential trial outcomes on pigeons' working memory. Animal Learning \& Behavior, 13, 103-108.

Sargisson, R. J., \& White, K. G. (2001). Generalization of delayed matching-to-sample performance following training at different delays. Journal of the Experimental Analysis of Behavior, 75, 1-14.

Sargisson, R. J., \& White, K. G. (2007). Timing, remembering, and discrimination. Journal of the Experimental Analysis of Behavior, 87, $25-37$.

Savage, L. M., \& Parsons, J. (1997). The effect of delay interval, intertrial interval, amnestic drugs, and differential outcomes on matching-toposition in rats. Psychobiology, 25, 303-312.

Trapold, M. A. (1970). Are expectancies based on different positive reinforcing events discriminably different? Learning and Motivation, $1,129-140$

Urcuioli, P. J. (1990). Differential outcomes and many-to-one matching: Effects of correlation with correct choice. Animal Learning \& Behavior, 18, 410-422.

Urcuioli, P. J. (2005). Behavioral and associative effects of differential outcomes in discrimination learning. Learning \& Behavior, $33,1-21$.

Urcuioli, P. J., DeMarse, T., \& Lionello, K. M. (1998). Transfer of performance to new comparison choices following differential outcome matching-to-sample. Animal Learning \& Behavior, 26, 139-153.

Wasserman, E. A., Grosch, J., \& Nevin, J. A. (1982). Effects of signalled retention intervals on pigeon short-term memory. Animal Learning \& Behavior, 10, 330-338.

White, K. G. (1985). Characteristics of forgetting functions in delayed matching to sample. Journal of the Experimental Analysis of Behavior, 44, 15-34.
White, K. G. (2001). Forgetting functions. Animal Learning \& Behavior, 29, $193-207$.

White, K. G. (2002). Psychophysics of remembering: The discrimination hypothesis. Current Directions in Psychological Science, 11, $141-145$.

White, K. G. (2013). Remembering and forgetting. In G. Madden (Ed.), APA Handbook of behavior analysis (Methods and principles, Vol. 1, pp. 411-437). NY: American Psychological Association.

White, K. G., \& Brown, G. S. (2011a). Reversing the course of forgetting. Journal of the Experimental Analysis of Behavior, 96, 177-189.

White, K. G., \& Brown, G. S. (2011b). Reversing the signalled magnitude effect in delayed matching to sample: Delay-specific remembering? Journal of the Experimental Analysis of Behavior, 96, 7-15.

White, K. G., \& Millar, J. G. (2014). Attenuation of the differential outcomes effect by extraneous reward. Behavioural Processes, $108,7-10$.

White, K. G., \& Sargisson, R. J. (2011). Maintained generalization of delay-specific remembering. Behavioural Processes, 87, 310-313.

White, K. G., \& Wixted, J. T. (1999). Psychophysics of remembering. Journal of the Experimental Analysis of Behavior, 71, 91-113.

White, K. G., \& Wixted, J. T. (2010). Psychophysics of remembering: To bias or not to bias. Journal of the Experimental Analysis of Behavior, 94, 83-94.

Williams, D. A., Butler, M. M., \& Overmier, J. B. (1990). Expectancies of reinforcer location and quality as a cue for a conditional discrimination in pigeons. Journal of Experimental Psychology: Animal Behavior Processes, 16, 3-13.

Wixted, J. T. (1989). Nonhuman short-term memory: A quantitative reanalysis of selected findings. Journal of the Experimental Analysis of Behavior, 52, 409-426. 